\section{Serge Valdinoci}

\section{François Laruelle : Pour une pensée-fiction}

Review Essay on François Laruelle's Tétralogos: Un opéra de philosophies. (Paris: Les éditions du Cerf, 2018).

Bionote: Serge Valdinoci is a French philosopher, author of numerous publications and, for the most part of his career, professor of philosophy at Université de Reims (Champagne-Ardenne).

F. Laruelle soumet à notre attention son Tétralogos, un Opéra de Philosophies. Le but qui est nôtre est de faire justice à l'endroit d'un ouvrage fondamental. L'enjeu est de grande importance, surtout à une époque saturée par les propos historiens-en philosophie précisément. En l'occurrence il importe de s'interroger sur le projet laruellien. L'Essentiel, ici, tient en deux mots. L'invention philosophique est le problème-clef. Telle est la force de cette question, totalement originale. Il est urgent de produire une Fiction, c'est-à-dire un processus qui crée en se créant, ou qui invente en s'inventant. Ce faisant nous reprenons la démarche des grands Romantiques s'exprimant après Kant, en lien avec Kant. Chez ces derniers il faut répéter que le souci d'une esthétique est patent. Et il s'agit de conjoindre alors esthétique et esthésique. Telle est la portée du concept de fiction. Chez F. Laruelle la théorisarion passe par ailleurs, du côté de la connaissance dont on bénéfice ou qu'on ignore. En ce sens et à notre avis, Laruelle échappe au nihilisme passif de nos contemporains. Mais Kant n'est pas adopté tout simplement. Car la fiction, selon F. Laruelle, est positive, et surtout pas fictive. Elle est fictionnante plus que nouménalement; elle invente dans un réel « écouménal » qui n'est pas ectypal pour autant. Avec Laruelle, nous quittons la psychologie des Facultés, et même celle qui plus tard sera reprise par Modernes et Postmodernes. La fiction, bien entendue, est de ressort créateur. Elle dit inventer du réel et ce dans le réel. Elle est de/ dans le réel. Esthésique et esthétique collaborent unement. Et ce encore en brisant la contemporanéité illusoire de la fonction sujet et de la fonction objet. Oui : le fictionnement invente en s'inventant, fait un effect dans l'affect. En termes laruelliens, une Tétralogie, ici très puissante, est un domaine de référence. Par exemple, il convient de dire avec force que la Philosophie non-standard ${ }^{1}$ touche à l'immense, alors que la Culture Europe, ou Culture Occident, compose sans broncher ni additivement, ni soustractivement. En résumé, la mesure projective, ou de mensuration, qui est effective, éloigne de l'immensité affective, tandis que la démesure, celle qui mord sur soi, s'effondre dans son soi, mais dans un potentiel non-philosophique, ou en Univers unionnant. Depuis son soi d'Univers, travaille un forcing, comme le conçoivent Cohen et Badiou. Ce dernier modélise scientistement le passage de l'Être à l'Événement. Chez Laruelle, on échappe totalement au scientisme malgré l'avis des tenants idéologisants de notre culture Europe. Nous pensons aux péri-philosophes de l'Institution, en France notamment.

Ici importe un effect un unilatéral, lequel pèse comme un forcing originé depuis I'Univers. La direction théorique s'instaure du « dehors » vers le «dedans ». Via l'idéologie de nos «penseurs » Européens, s'installe un grand frémissement mystique en europe, lequel abandonne l'esprit de nos contemporains ratiocinants, appuyés sur I'hypokeimenon fallacieux du sujet-substance, quand bien même il serait déconstruit (par Derrida). Sur ce point, Laruelle est intempestif. Il bouscule irréductiblement. C'est un théoricien du Passage définitif, et qui ne passe pas dans le Tétralogos. Entre Socrate et la non- philosophie, il y a un Milieu immense d'univers qui n'est pas graduable et auquel on réfère en intuition, ce bain de Sens sensible. Il y a immersion.

Notre effort conséquent se structurera en vertu d'une économie intrinsèque, ou en-ergiquement im-manante. Voyons bien que cette en-ergie n'est pas industrielle, c'est -à-dire énergique. Car l'économie n'est point inscriptible dans l'espace-temps. Elle ressortit à une spatiolytique qui permane mais qui est saillante jusqu'aux tréfonds.

${ }^{1}$ Sachons qu'un standard, conforme à une norme de fabrication- ne produit rien créativement. II se produit sans créer. En d'autres termes, un standard ne tolère que la rémanence spatiale de la pensée philosophique artificielle, au sens bergsonien de la locution. Mais il y a mieux. En ef-fect, la pensée Philosophie emprunte abyssalement au réel d'écoumène, mais en le déniant culturellement. Les grands historiens de la philosophie cherchent un standard, jusque Heidegger y compris. Mais l'opération est vaine. La problématique conceptualisante cache en réalité une mystique -une mystique pensante, ou presciente hors toute standardisation culturelle. 
Avide d'un intrinsèque, $F$. Laruelle donne force immanente à une pensée non technologique, non industrielle afin de transsubstantialiser l'espace - temps via un enspace autolytique et per-manant. Mais sans doute notre non-philosophe préfèrera travailler sur un substitut intéressant. En l'occurrence sont élues la Musique et la Philosophie. Et cette opération est impérieuse pour mener à bien une Philosophie non-Standard. Ainsi si vaut une Philosophie qui est la plus belle des Musiques, alors on remarquera que s'impose - sans Socrate - la musique. Celle-ci est la plus belle des Philosophies - selon le non-Philosophe.

Mais allons plus avant. Les deux nécessités (Philosophie-Musique) dessinent, quand elles sont co-examinées, un afigural immense ou interne. Ou bien un im-manant aboutit à individualiser (en immanence) une maison, pour habitation, un Oïkos en qui s'assume une Révolution messianique fondamentale. Nous avons affaire à un enspace (et non pas espace philosophique). C'est l'enspace du réel écouménal, son immensité intense. Le texte laruellien nous aidera à progresser. Mais, en l'invention il convient de ne pas questionner vainement. Nous sommes abonnés à une tâche qui ne ressortit pas au commentaire. Notre aventure est ici de questionner en l'interne immanant. Dès lors, notre tâche est assez simple. Force est d'accompagner la problématique de Laruelle en la respectant, et en amplifiant sa donne expressionnelle par un impressionnel et proche et lointain.

Pour commencer d'argumenter, d'expérimenter dans l'interne, convenons du fait que la Philosophie - si emportée dans sa suffisance Culturelle - est supplantée par un forcing dont F. Laruelle procède, mais travaille pour le transsubstantier radicalement. II en est ainsi : chez Cohen et Badiou le forcing est "idéalisé ", et donc modélisant. A notre sens, le non-Philosophe est autonome, radicalement. Il échappe au modèle Badiou. Disons rapidement qu'un unilatéral fondemental juge de la situation. Le philosophe procède « intentionnellement ", il part de la situation de la Pensée culture, ou pensée Occident. Telle est la schématique de la pensée Philosophique : I'allée du dedans au dehors. Quant au non-Philosophe, il procède de la « direction » inverse contraire. L'Univers est premier, et le philosophe hallucine dans l'attitude cognitive. II « oublie » le « frémissement » mystique dont la culture Philosophie n'est qu'une dénégation dilatée. Ainsi, nos Modernes ratiocinants ne recèlent qu'un hypokeimenon fallacieux : le Sujet- substance. Ainsi Laruelle est intempestif. C'est un théoricien du " passage ", qui, pour son propre compte, est soustrait au passage. Entre Socrate- Platon et la non- Philosophie, il y a un milieu « infini » d'univers immense et non pas d'Univers graduable, - en qui on rétroréfère scientifiquement. Car la rétroréfèrence immanente, est en intuition. Elle n'est pas quantifiable. Le théoricien penseur baigne en l'immanence et se mesure-en l'intuition omni-préséante. II s'agit ici de bien commencer, en repérant la structure de la préséance en Culture. Depuis les Modernes, le Philosophe utilise le Discours du Monde en tant qu'a priori. De la sorte, le dimensionnel gnoséologique intervient dans le processus culturel initial. Ce dernier est toujours déjà épistémologique, et s'inscrit dans la dualité parole -chose. Mais une difficulté apparaît. Dans le régime épistémologique, on dira avec regret que la philosophie est dans le siècle, ou ne prend comme arme de connaissance que des idées générales, ou bien compilées. Telle est absolument la nouvelle donne. A titre simplement indicatif il convient d'énoncer que toute une pratique - sémantique - doit au moins trouver place ici. Car le « rendu » syntaxique mérite de se voir contrôlé fermement. Les sciences dites exactes se savent dépendantes du "sensé ».

F. Laruelle n'ignore pas la difficulté. Existe un Nuage d'inconnaissance. Ceci signifie que le nuage - ou sfumato - dans notre « prise »de/sur les choses elles-mêmes, échappe principiellement à son exhibition en Culture, sous l'espèce de la connaissance. La chose est entendue. Mais de la sorte il se manifeste que la fondation sans connaissance renvoie à un phoros (un porteur). Vaut une doxa primitive (Husserl), un proto-phoros (une protophorique).

Alors résonne une alliance, une créatrice. Un Tétralogos laruellien ex-prime sur trois paliers (générique, quantique, philosophique) et philo-musical, les lois argumentées dont la puissance fait Impression tout en impressionnant le lecteur par la force d'exposition du propos laruellien, tout à fait nouveau dans la société de nos Philosophes universitaires. À notre avis, et comme toujours chez F. Laruelle, il convient de " redimensionner l'immensité » des textes et ce à des fins pédagogiques. Nous savons bien, à titre prolégoménal, que en interne, le proche est lointain. Mieux, le tact est irremplaçable, fût- 
ce par le contact, ce dernier organisant le propos de toute démarche dans les sciences scientifiques. Ainsi tout contact se prélève sur le tact spatiolytique. Ou encore, Bergson aura montré définitivement que l'intelligence est abstraite, ou artificielle. Elle intuite dans les choses elles-mêmes (Husserl), mais n'articule aucunement dans le «nuage d'inconnaissance », comme le dira F. Laruelle.

Prenons un exemple par ailleurs. Une théorie naïve de l'homme, cet instantané s'autolysant, omet malgré tout la Nature franche de l'homme en tant qu'esthèse en intuition. Ici un « plein » autolytique travaille à même l'homme même. Et pour traiter de celui-ci (cf. Eckhart) on dira que celui-ci est esthèse protopathiquement pan-analysable, étincelle divine logeant en l'âme. En somme, pour échapper à la glaciation normopathique en l'état contemporain (au XXème siècle), de deshérence Culturelle il est exigé d'inhérer, d'assumer que la connaissance de l'homme est encore dans I'homme.

En deux mots, connaître « $X$ « c'est faire objet en lui. Mieux : c'est naître de lui en lui. Et c'est vrai, dans ce contexte sur-analytique il est difficile de mener à bien une science de l'homme en l'homme. De notre " côté « un futur Guide des égarés devra recourir à une pathique, à un concept endoceptant et un endocept concevant. Ce faisant intervient alors l'Autre de l'effectuation métaphysique en Europe. Nous nommons ici : affect, cet Autre en Occident. Ces deux instances construisent une Pensée une, pensée qui fut réprimée par la Culture Occident. Cette Culture, d'ailleurs, est loin de baisser son pavillon. Au contraire elle sature son territoire " sauvage ", et immobilise la pensée comme mouvance, dira Bergson à sa manière. Nous saluons F. Laruelle très tranquillement : civilisationnellement, c'est une vaste entame de la Culture captée par un réel écouménal. En langage husserlien bien accompli écrivons que cette entame est sondée opératoirement et non thématiquement. Ici travaille l'invention créatrice, ou fiction. II reste à savoir comment.

En tant que laruellienne, s'offre une grande aventure qu'il nous est possible de caractériser. Un vécu sans vie fait office ici. En effet, dans la donne (représentationnelle) d'une science scientifique, il s'avère que la « vie » brute, immense à sa façon est incommensurable au « vécu » qui renvoie - mais abyssalement - à un domaine immensionnel, ou à l'immédiat vif. Dès lors, se précise un chemin : dans une science humaine - et in-exacte scientifiquement, l'ordre du contact se mêle à celui du tact et les deux approches opèrent par interaction de l'immédiat et du médiat. Alors tout fait difficulté, car opère le brouillage indélébile. La science opérationnelle abrite, certes dans le faible, une difficulté forte. Dans le centre du brouillage, opère un proto-logos, c'est-à-dire ici un Proton pseudos sub-phénoménologique. Dans ce contexte, Merleau-Ponty nous dira que la Perception enferme un trouble en tant que Proton. Poussons plus loin : dans une science dite humaine s'exhibe un brouillage. Immédiat et médiation s'enveloppent et se développent, et ce dans un en-space per-manant. L'idée d'un proton, mais Proton pseudos s'impose. En effet, l'origine (immédiat) et l'originaire (médiation) se « confondent polémiquement ». La perception effectuante et extrême est affectée dans une déception extrême et réciproquement. Un Monôme abrite un polynôme en tant que Proton pseudos. Ce dernier éclate par enclatement, ou épaississement.

Mais sachons aller plus avant, ou sachons fictionner. Ceci-dit, il est fallacieux de réduire le « comportement » à une structure, à une syntaxe coordonnée avec une sémantique, ce que tente pour sa part Goldstein. Mais ces penseurs philosophes sont arrêtés par l'état de la Biologie. En effet, les biologistes précèdent les Philosophes d'une génération au moins. Ceci fait que ces Philosophes sont intrinsèquement en retard, qui plus est dans leurs attitudes nocturnes semblables à celles des oiseaux de Minerve.

Si les Philosophes sont en retard sur le corpus biologique, le non-Philosophe construit un espace se spatiolysant. Qui plus est, et dans cette voie heuristique, le passage des représentations a des présentations - chez Laruelle - ne cesse de faire difficulté. Autrement dit, une héroïque est à penser dans l'immanence. Et F. Laruelle, nous le savons n'entre pas dans la question de la protophorique, celle d'un porteur premier. Deux portes semblent se refermer catastrophiquement. Cependant, il convient d'entrer dans ce qui fait autorité chez les Philosophes, outre l'exigence fondamentale de la non-philosophie chez Laruelle. Outre l'impossibilité de la jonction de la représentation et de la présentation, nous avons peu à peu compris que le Phaïnomenon est une Krisis de/dans les hommes, laquelle se produit avec la nécessité irrègulière d'une pathique déployée en des pathétiques culturelles. Nous pensons à la pathologie médicalisée etc. 
Par exemple, une héroïque théorétisation forte ou zigzag anagogique, interne, prend sens véritable. Ceci nous réengage, malgré tout, dans un géométral d'écoumène, ou sans fossilisation géométrique. Dans notre exposé visant Laruelle, on dira dûment qu'un altéral fictionnant permet d'admettre qu'une théorie de l'homme, qu'évoque avec grande précaution F. Laruelle ne concerne l'homme qu'en tant que cet homme est altéral, en crise, faisant marge de luimême. L'homme en fiction est une chaotique de crise, disons : de crise " positivante ", mais qui n'échappe au chaos que si l'en-cahot est sécurisé, protégé par nombre d'interventions culturelles. Alors le fictionnel engage dans une écouménalité créatrice. Quant à la Philosophie, elle ne produit que des «fictions " superficielles, tout au plus commodes et jouant leur rôle de stabilisateurs, de compensations psychiques, socialgique - au sens médical de l'expression. Certaines compensations œuvrent en sourdine. Ainsi la normopathie devient, à notre époque, suressentielle. Et le Normal juge de la Norme. De la sorte, nous saisissons mieux ce qu'est une fiction créatrice. Laruelle ouvre une grande route. F. Laruelle est bien un Passeur qui, riche de sa noble matière première (l'immanence) ne subit pas les lois délétères du passage. Dans la suressentielle pauvreté philosophique - celle du standard aplani - le non-philosophe travaille en musique, elle qui fausse la première place aux Analytiques et ce dès Platon annonçant une loi d'harmonie musicale fondant la théorétique discursive. En initiant la richesse du débat, Socrate énonce que le Philosophique est la plus belle des musiques. Mais reste concurremment que la musique est la plus belle des Philosophies. Nous sommes pris dans un cercle vertueux, l'équivalent d'une boîte noire. On retrouve ici l'idée d'un encart qui fait écart, et bien sûr d'un écart qui fait encart. Le tout travaille en épaisseur, et produit des reliefs d'enfoncement et de défoncement. Telle est la Krisis en interne.

Mais la production d'Harmoniques à structure musico-philosophique ne suffit pas, malgré le propos verticalisant. En musique tonale, il y a de l'horizontale ou contrepoint. Chaque instance est rétroréférence. En ce sens, convenons - mais heuristiquement seulement - que le pivot harmonique est congruent avec les élancées contrapuntiques. Alors, ce pivot accordé à l'immense, fût-ce par contact ou éloignement est d'ordre pathique et aucunement pathéthique. L'opus est ici un itinéraire se développant internalement dans une téléologie immanente. La puissance de notre non-Philosophe correspond à un é-norme embarquement en tant que Fiction, certes à intra-structurer internalement. Alors énonçons dans l'immense le discours impeccable de l'Affect en culture bouleversée. Nous ef-fectuons le trajet mesurable qui se mesure à l'immensité. On parlera de deux instances encastrées (l'effectuation et l'affectuation). Celles-ci se superposent, comme le disent nos physiciens contemporains. En ce sens, on accède par fictionnement à un large Hypokeimenon ; celui-ci est de type deux, et laisse à son sort désuet le principe de substance aristotélicien, lequel est enveloppant dans l'esprit d'un tact immensément immanent. Il est vrai que le concept de pathique, hors toute pathétique Culturelle, offre l'occasion d'œuvrer interactivement tact et contact. En ce cas, l'itinéraire musicalisant-musicalisé, enfin ramené à lui-même même, aidera à penser en dedans de l'univers non-métrique- qui n'est point I'Univers scientifique. Voilà qui signifie promouvoir une fort large «pensée- invention » via une askesis en tant que contemptrice du Proton pseudos. Cette invention est de ressort d'une en-ergie comme accentuation (ou accentuation). N'omettons pas ici que l'énergétique intra- spatio-temporelle est ordonnée au principe d'en-ergie. Celleci est de ressort mystique. En somme l'expérienciation de type mystique, qui n'est point connaissance - nous le savons bien - est celle d'une démarche performante. En l'occurrence les significations référentes retournent à la puissance interne du Sens. L'opération relève d'une spéculation en-ergique. Dans son langage, Husserl évoquait l'identité du Sens et de la signification. A Husserl fait défaut le travail sur en-ergie et énergie. Certes, Husserl ne possède pas le concept d'idée - force, que Nietzsche place en chaire, sans doute précipitamment. Ici personne ne voudrait prendre le relais. Toutefois, F. Laruelle permet de penser plus avant. Husserl cherche à bâtir une Anthropologie transcendantale, et cela en vain, assurément. Tout le XXème siècle bien-pensant cherche en vain faute d'avoir reconnu le Proton pseudos, ou première déception, qui est perceptive. En fait, notre approche consiste à structurer cette déception sans nous perdre en elle. Au vrai, il convient d'étudier en l'interne, hors l'efficace perceptive qui est désorientation vive. Grosso modo, la tâche consiste à penser en le réel écouménal en logeant chez lui. De son côté, Laruelle nous convie à sonder l'écoumène en tant que Lebenswelt. Naître en lui est plus opportun que connaître de lui. Ou 
encore, il convient de maintenir la force de la pan-analytique non Européenne. Dans ces conditions, une théorie irruptive fait intrinsèquement - retour sur soi - et ceci sans s'adonner à la réflexion philosophique. Alors l'intrinsèque est roi. C'est un viscère d'univers en-ergique en temps qu'en dedans de l'immensité en-ergique. F. Laruelle est un promoteur vaillant. II écrit hors le XXème siècle. Nous souhaitons avoir travaillé dans la crise-Europe, en sachant que nous procédons de la Krisis- europe, et de la Terre-univers. Car la reconnaissance du monde de la vie rétroréfère à la Terre des hommes. Le non-philosophe prépare une intra-structure vivant et initiant, pure dans sa Maniera inconditionnelle. L'Européen apprend à habiter la spécificité d'europe. L'européen est tact en action, et précède la rigidité des scientifiques, de plus en plus culturalisés et inventant des contacts pour procéder Culturellement. Ainsi la question du partage des connaissances est tout un art, à partir d'une aisthesis dont la portée est à contrôler.

Oui : d'abord il convient de placer la problématique de l'aisthesis. Quant au lecteur, il analyse celle-ci tout en procédant d'elle. Nous sommes en présence du phénomène de zigzag où affect et effect s'échangent, se combinent viscéralement et unement. Le zigzag qui fait sfumato (l'enfumement enfumé) se dit des choses elles-mêmes. On en parle alors que, concurremment, il est parlé depuis lui. L'équivoque est totale, sans être hallucinée par la pirouette hégelienne, si habituelle. Prise dans ce mouvement de pensée inventionnelle, la pensée europe symbolise en étant symbolisée, et ce indéfiniment.

En temps qu'inventeur brut F. Laruelle force (forcing) la Philosophie depuis l'univers adsolu et im- manant qui n'est pas celui des scientifiques (ou Univers). L'univers du Phaïnomenon échappe à son encasernement dans I'Univers absolu des cosmologues contemporains. L'univers adsolu est base de rétention, ou ce qui fait préséance. On le voit, il manque ici au moins une théorie de/dans la Culture qui éloigne de tout freudisme dégénérant en culturalisme mal contrôlé. En somme, le zigzag n'est point relevant d'une traçabilité - Monde -, laquelle fait en définitive partie du Monde. Le zigzag progresse dans l'intuition massive universelle. II sous-traite l'intuition, mais s'emplace en cette dernière. Il fait bien de/dans.

F. Laruelle invente en dé-couvrant le chaos comme sous- sol du Kosmos. Hors le XXème siècle révolté on trouve un Drame total, une terreur incommensurable dans le Kosmos. Ce dernier est hypersensibilisé, débordant. Un quasi-système de la douleur trouve place, dans la seconde partie du siècle... M. Henry est tenté par l'aventure d'une Af- fectivité. Cependant, l'affectivité n'est pas pensée vraiment comme L'Essence de la Manifestation la revendique. Sauf à généraliser dûment la Manifestation, qui échappe intrinsèquement à toute " monstration ", intentionnelle, on dira à juste titre que la Manifestation se dit et se redit en interne. La Manifestation est auto - affection, mais le Verbe henryen se reprend interminablement en écrasant sa propre pertinence. M. Henry prend dans une source absolve, mais en taisant son subterfuge. La maniera refait maniera et ainsi de suite. Henry prend à l'Entre ou à l'immanence. Ainsi, la maniera l'emporte, bien qu'elle soit de seconde main, ou s'installe dans le Milieu qui absorbe l'adsorbé. Par-delà la débauche des citations que Henry emploie pour parvenir à ses fins, Henry "incide" vers une coïncidence de l'adsorption et de l'absorption. Henry ne parvient pas à ses fins. Cette Incidence jamais aboutie indique que la " théorie » flotte sans rémission. Henry «flotte " à son tour, cherche un transcendantal théorisant, mais toujours en vain. Seul Un Dieu " ferait » l'affaire. Voilà que M. Henry nous entraîne à la recherche éperdue d'une merveille, quand flotte l'esprit qui ne progresse aucunement. Dans son Tetralogos, F. Laruelle, lui, met en place une vraie maniera, quand la merveille fait masse avec autonomie. En ce sens, se propose un étagement en profondeur réelle, cette foisci. Laruelle écrit en immanence. Pourtant, sa Philo-fiction n'est pas élaborée. La réalité est que le non-Philosophe constate que cette Philo-fiction n'est pas immanence, même si cette dernière est invoquée /évoquée. La raison de cette théorie renvoie à une théodicée largement sous-jacente. F. Laruelle remplace I'X situé entre le discours et le Monde par une "théorie sourde ", un divin immanant. Le respect est de formuler cet ordre « sourd » mais fort riche pourtant. Il existe une théodicée immanente, prenant forme dans les théories à former, à formuler. Dans en ce cas, le rationalisme "laruellien » propose l'idée d'un telos immanant, ou Divin si l'on veut parler au plus simplement. Ainsi le Tétralogos assume une pensée toute particulière : il existe bien une pensée Laruelle, celle d'une non-philosophie fictionnant écouménalement en tant que de/dans l'altéralisé. Ceci amène la remarque suivante : enfin le Tétralogos est moteur, hors la trilogie philo-logique attendue. Ainsi l'analytique, bien en- 
tendue, suscite au vrai une maniera développée en une altéralisation qui vaut l'unilatéralisé utilisé par notre non-Philosophe. Selon nous, le réel écoumenal prend enfin son intérêt. Il " généanalyse » le réel dans ce réel lui-même, européen. Alors il faut dire que l'aisthesis est contrôlée, et cela une dernière fois. Elle est le fil rouge indélébile, la Figura serpentinata qu'amplifie la majesté encore statique de la Philosophie non-standard. Désormais la ligne dite indélébile fait « ergon » flexueux ou serpentin qui ouvre brèche ou même fait brèche en elle-même. En l'enspace, l'ergon s'aventure au loin et s'en revient en-ergiquement ... pour repartir. Mais tout n'est pas dit. Reste à fictionner fondamentalement, à faire colimaçon dans I'univers mouvant et à œuvrer pour susciter entièrement dans le non-espace-temps, une figuralité immense - altéralisant formes et formulations -. Pour ainsi méditer, nous avons plongé dans l'immanence staturale, puis travaillé dans le Kosmos pour le servir. Car la situation d'homme, bien entendue, ne pèse pas. Elle fait braise, ou allume I'homme loin des Humains et leur psychologie, et de la socialgie qui est écrasante. Ainsi, une théorie de l'Humain - toujours - en - Culture est renvoyée à l'homme hors les dimensions qui en appellent à l'Humain en Culture. Il en va de l'incommensuralité de l'homme en tant que " sauvage " radical et dépositaire de sa situation comme origine. Mais prenons attention une dernière fois encore : pour F. Laruelle la Philofiction est base de créativité. Cette fiction, prise dans son identité indiscutable, renvoie à l'origine nue, hors la recherche super-appareillée d'un originaire légiférant (cf. Kant, qui accentue cette attitude). Dès lors, en l'acte créationnel émerge une origine en tant que brute. Mais la recherche, en immanence, ne saurait trouver son aboutissement dans la présentation d'une donne brute de la pensée-en-immanence. II faut, en la circonstance, commencer de parler plus avant. Car ceci renvoie à la pensée-espace «lysée », ou spatiolysée. De la même manière, la pensée-temps se met à permaner irréductiblement. Dans ce contexte « tout se retourne ». Réduction et donation s'échangent identitairement. En création, imploser est exploser, et réciproquement. Mieux : en la transcendance "transcendantale", le Discours du Monde est à élaborer. En immanence en revanche, le discours est monde. Tout est dit.

Dans ces conditions, la Fiction créatrice est lourde du statut du «monde », lequel est transsubstantiable. Alors, il faut dire que la
" conversion » du Monde en monde, et inversement, ressortit à un processus mystique élaborant. Dans cet ordre d'idées, il nous reviendra de penser la Lebenswelt (le monde de la vie) dans toute son immensité, ici transphilosophique. Nous aboutissons, en ce sens, à majorer la «trouée » térébrante et en-ergique, ou la fonction-univers qui « verticalise" (Cf. Heidegger et Merleau-Ponty). Mais sachons, en tout état de cause, lire opportunément F. Laruelle. Ce dernier nous donne accès, avec son Tétralogos, ou propos toujours initial, originant. Celui-ci demeure accroché dans l'immanence radicale et conséquemment une non-philosophie inclut la philosophie.

La philosophie ne rend pas compte de son Verbe. Elle réfère à l'immanence qui implique une expérienciation ou expérience au cœur des choses elles-mêmes. Il existe une pragmatique qui utilise la thèse philosophique en Culture. D'où une reprise est nécessaire. Quelle est au juste la fonction de l'immaner? La non-philosophie « prend » sur celui-ci ou le rétrograde mais seulement heuristiquement. Une philosophie non-standard impose un propos indélébile, car il convient de reproblématiser. Ce dernier propose de procéder du commencement, comme il a déjà été dit en physique quantique où il y a superposition - celle de l'affect et de l'effect. Il y a difficulté car chez F. Laruelle l'affect n'est point moteur. L'effect est Maître dans la maison (Ö̈kos). Dès lors, il s'impose de refictionner en produisant un skhema d'univers qui ne soit pas une copule d'Univers. Ainsi, la diplopie de l'affect et de l'effect unifierait affect et effect. En conclusion cette approche du fictionnel, en-ergique, formerait une structure intêressante. À nouveau le Tétralogos (F. Laruelle) emplit la pensée qui invente immédiatement en l'univers principiellement affect. Cette pensée-masse court-circuite les dimensions toujours déjà figées. Les effects sous-traitent les affects dans le Monde de l'espace-temps. C'est pourquoi, le rapport laruellien entre musique et philosophie est une Donnée (un datum). II conviendra de resituer musique et philosophie, hors leur datum de pensée espace-temps. II s'impose, on le sait, d'échapper à la substructure pour la recentrer en spatiolyse per-manant. Pour entrer dans la compréhension, certes, extérieure, de la situation théorique, référons-en à la métaphore, certes pertinente. En Biologie nous dirons que la diplopie est ce phénomène dans lequel la perception de deux sources lumineuses est nécessaire pour la perception d'un seul objet. C'est une situation 
pathologique. En quel sens europe est-il partie prenante? Allons au plus près. Dans la pertinence métaphorique, comment ne pas remarquer une homologie ? Ici, Philosophie et Musique sont co- impliquées dans l'Entre-deux. Dans cet Entre - qui n'est pas l'Autre des différentialistes contemporains, l'interaction est une première donne. Et le fond commun de cette donne-datum est l'espace-temps, ou le même à même lui-même, c'est-à-dire répétition pure ou articuler minimal. En ce cas, une pensée artificielle, ou ordinatique quasi philosophique, suggère un écart fort entre Philosophie et Musique. II y a en somme interaction. L'Entre est rigidifié.

Mais une pensée-europe est tout à fait différente. Ici Philosophie et Musique sont autrement qu'interactifs. Ils sont intractifs. Musique et Philosophique se fondent l'un dans l'autre, ne com-posent aucunement. La diplopie a cessé de valoir, elle fonctionne comme accélérateur vif, dynamisme energique. En vérité, et désormais hors métaphorique, l'Entre est en l'aisthesis formidable ou domaine $d^{\prime}$ Intuition. C'est dans ce phénomène que Musique et Philosophie s'abandonnent l'une dans l'autre, et baignent en aisthesis. En ce sens la copule prédicative est une illusion dont la force dynamique provient d'une non-prédication fondementale, et pas seulement fondamentale.

La puissance du travail de F. Laruelle est indiscutable. Et elle engendre une grande Pensée. En effet, la Philosophie-non-standard donne à commencer de penser créativement le réel dans le réel. Elle délimite en ce dernier et respecte son sacré. Elle est ef-fective absolument. Notre Pensée europe, ou Pensée sauvage, se laisse animer par les balances d'univers. Elle donne consistance à une empathie universelle, et endogénétique. Avec d'autres mots, elle s'approprie le nerf écouménal - qui est sien -On peut encore dire ceci : I'écoumène fait flamme de soi et ce en af-fect. Sans la basis écouménale, il convient d'admettre que l'intuition d'univers se démettrait de soi. Elle resterait une carcasse inanimée, sans accélération intense, ni Loge irréductible... mais désert à tous les vents et désolations. En la théorétique bien entendue, un « $X$ » pense en agissant affectivement, et ce sans la médiation traditionnelle par la fonction « connaissance».

Ainsi un pur théoricien vif laissera se creuser le Monde en monde du/dans le logos - et non pas Logos. La vérité est analectique et non pas dialectique. Dans cet ordre d'idées, la pensée - invention fictionne. Elle se propose sans vesture occasionnelle. Elle bombine par spasmes per-manents. Mais le chaos n'est qu'apparent. En effet, l'affect ne se referme aucunement sur soi, pas plus que, sur un territoire subjectif toujours ensanglanté. L'af-fect brûle dans sa généralité. De ce foisonnement, ou sfumato général sont empreintes impressionnellement les localités af-fectives expressionnelles - celles des sciences scientifiques par exemple-. Le travail dans ces dernières est positif, indéniablement. Cependant, il est encadré culturellement. Ce sera la tâche de l'europanalyse que d'ouvrir la porte au sauvage, mais en n'omettant pas le rôle fondemental de la dynamique en-ergique. Car n'oublions pas l'Essentiel, voire le Sur-essentiel : une pensée mystique hors l'Europén, ou l'Occidental, ne connaît point d'abord. D'abord, elle co-naît au monde de la vie - comme aura dit P. Claudel, mais sans expliciter plus. Au départ est l'Af-fect en qui s'activent les ef-fets de savoir. Telle est la non-Philosophie, notamment dans les débuts de sa méditation, dans les premiers ouvrages. La spécifité de l'europanalyse est de « commencer » en af-fect mais en y demeurant définitivement. L'épaissement, les arrières-plans anti-tautologiques - ou solides-, forment le sous-sol de toute théorétique bien consistante. Ainsi, l'affect, qui ne trompe point, demande un discours intrinsèque, ou en immanence pour que soit évitée la mise en Culture. Reste la puissance de l'immaner, puissance dicible qu'invoquent Non-Philosophie et europanalyse, mais chacune dans sa maniera à respecter scrupuleusement. 